\title{
The effects of unequal reward distributions on cooperative problem solving by cottontop tamarins, Saguinus oedipus
}

\author{
KATHERINE A. CRONIN \& CHARLES T. SNOWDON \\ Department of Psychology, University of Wisconsin, Madison \\ (Received 17 January 2007; initial acceptance 13 April 2007; \\ final acceptance 18 April 2007; published online 14 November 2007; MS. number: A10674)
}

\begin{abstract}
Cooperation among nonhuman animals has been the topic of much theoretical and empirical research, but few studies have examined systematically the effects of various reward payoffs on cooperative behaviour. Here, we presented heterosexual pairs of cooperatively breeding cottontop tamarins with a cooperative problem-solving task. In a series of four experiments, we examined how the tamarins' cooperative performance changed under conditions in which (1) both actors were mutually rewarded, (2) both actors were rewarded reciprocally across days, (3) both actors competed for a monopolizable reward and (4) one actor repeatedly delivered a single reward to the other actor. The tamarins showed sensitivity to the reward structure, showing the greatest percentage of trials solved and shortest latency to solve the task in the mutual reward experiment and the lowest percentage of trials solved and longest latency to solve the task in the experiment in which one actor was repeatedly rewarded. However, even in the experiment in which the fewest trials were solved, the tamarins still solved $46 \pm 12 \%$ of trials and little to no aggression was observed among partners following inequitable reward distributions. The tamarins did, however, show selfish motivation in each of the experiments. Nevertheless, in all experiments, unrewarded individuals continued to cooperate and procure rewards for their social partners.
\end{abstract}

(c) 2007 The Association for the Study of Animal Behaviour. Published by Elsevier Ltd. All rights reserved.

Keywords: cognition; competition; cooperation; cottontop tamarin; mutualism; reciprocal altruism; reciprocity; Saguinus oedipus

There is no universally accepted definition of cooperation. Some researchers have focused on the behaviour of the individuals, and define cooperation based on the ability of actors to coordinate their actions (Boesch \& Boesch 1989). Others have concentrated on the distribution of costs and benefits, and defined cooperation as any act by one individual that benefits one or more other individuals, regardless of whether any coordination in action occurred (Hamilton 1964; Sachs et al. 2004). Interactions that result in immediate, equal benefits for all actors are categorized as mutualism or by-product mutualism. In contrast, reciprocity or reciprocal altruism takes place when repeated interactions between individuals result in equal benefits distributed over time (Trivers 1971; Mesterton-Gibbons \& Dugatkin 1997). Here, we use a behaviour-focused definition in which cooperation occurs when two or more individuals combine efforts to achieve a common goal, and

Correspondence: K. A. Cronin, 1202 West Johnson Street, Madison, WI 53706, U.S.A. (email: kacronin@wisc.edu). we examine the effects of various reward distributions on cooperative performance.

Numerous experimental studies have examined cooperative problem solving in animals (reviewed in Dugatkin 1997; Tomasello \& Call 1997; Noe 2006; Silk, in press). However, as noted by de Waal \& Davis (2003), few studies have involved systematic manipulation of economic variables such as the relationship between the effort required to solve a task and the allocation of rewards to actors. In one such study, pairs of capuchin monkeys, Cebus apella, were required to combine efforts to pull a weighted tray of food towards themselves. The capuchins showed lower cooperation success when presented with a tray baited for only one actor when compared with mutualism trials, despite food sharing being common following trials in which one actor was rewarded (de Waal \& Berger 2000). Hattori et al. (2005) showed that capuchin monkeys, C. apella, successfully performed complementary actions for mutual rewards, and then continued to cooperate but at a lower success rate when only one individual was rewarded on each trial. In these studies, the experimental 
design allowed both actors to gain benefits within a test session. Additional research is needed to determine how extended delays between reciprocation (i.e. across days) are tolerated by the actors and what effects the extended delays between reciprocation have on the cooperative performance.

The physical distribution of rewards in space may also affect cooperative behaviour. Greater cooperation success may be obtained for dispersed rewards than for clumped rewards (capuchins, C. apella: de Waal \& Davis 2003; chimpanzees, Pan troglodytes: Melis et al. 2006b). This effect may be because of dominance interactions or more generally the degree to which payoffs appear 'guaranteed' to the actors. Presumably, when the probability that an actor will benefit decreases, either because rewards will be monopolized by a dominant individual or because the chance of obtaining rewards in a scramble competition is less than when rewards are delivered directly to actors, the likelihood that the actors will exert a cooperative effort also decreases because the potential cost to the actor increases.

Primates may be averse to inequitable distributions of rewards. Brosnan \& de Waal (2003) indicated that female capuchin monkeys, C. apella, refused to accept rewards of lesser quality than rewards received by nearby conspecifics. This effect was exaggerated when the recipient of the higher-quality reward was required to provide less effort than the subject presented with the lower-quality reward. Humans also show aversion to inequity in ultimatum games, in which participants typically reject proposals by other players to split shares below $25 \%$ of the available amount and instead opt to receive nothing, even though this action does not maximize the participant's self interest (reviewed in Fehr \& Fischbacher 2003). Roma et al. (2006) argue that the apparent aversion to inequity shown by Brosnan \& de Waal (2003) may be explained by frustration due to an individual's past history with greater rewards (but see Brosnan \& de Waal 2006). Regardless, either explanation for refusal of rewards (inequity aversion or frustration) would suggest that actors would be less likely to cooperate for unequal reward payoffs than for equal rewards.

In addition, the social characteristics of a species, such as their degree of tolerance for nearby conspecifics and characteristic amount of behavioural coordination, may influence their ability for both social learning (Coussi-Korbel \& Fragaszy 1995) and cooperation (Cronin et al. 2005). Trivers (1971) noted that in species with strong dominance hierarchies the likelihood of reciprocal altruism is reduced (in this case reciprocal altruism refers to the distribution of rewards regardless of coordinated action). Presumably because of high levels of intragroup competition in Guinea baboons, Papio papio, Japanese macaques, Macaca fuscata, and rhesus macaques, Macaca mulatta, these species did not coordinate efforts to move heavy baited stones (Fady 1972; Burton 1977; Petit et al. 1992), whereas Tonkean macaques, Macaca tonkeana, characterized by less-strict dominance hierarchies and greater social tolerance, were more often successful at coordinating their actions to displace the baited stone (Petit et al. 1992). Consistent with the idea that socioecological context can predict performance on cooperative tasks are the observations that chimpanzees performed better in competitive than in cooperative paradigms (Hare \& Tomasello 2004), and that chimpanzees do not choose to donate rewards to conspecifics even at no additional cost to themselves (Silk et al. 2005; Jensen et al. 2006).

Beyond the general social characteristics of the species, the nature of the relationship between the specific individuals faced with a cooperative task may affect their ability to cooperate. As argued by van Schaik \& Kappeler (2006), individuals bonded over an extended length of time likely do not evaluate the immediate costs and benefits of their behaviour, but rather the long-term benefits and costs exchanged throughout the relationship. Dominance asymmetries may also affect cooperative success, either in the form of coercion by dominants to solve the task or avoidance of the task by subordinates (chimpanzees: Chalmeau 1994; Chalmeau \& Gallo 1996; orangutans, Pongo pygmaeus: Chalmeau et al. 1997; keas, Nestor notabilis: Tebbich et al. 1996). Werdenich \& Huber (2002) showed that success on a task requiring common marmosets, Callithrix jacchus, to coordinate behaviours in asymmetric relationships was contingent upon the dominant individual receiving the rewards.

In the present study, we examined the effects of different payoff arrangements on the cooperative behaviour of pair-bonded cottontop tamarins, Saguinus oedipus. Cottontop tamarins are an ideal species for investigating cooperation as they are socially monogamous cooperative breeders where both mature offspring and fathers assist in infant care by carrying and sharing food with younger offspring (Snowdon 1996). Tamarins coordinate behaviour for infant care, travel, food sharing and predator detection (reviewed in Caine 1993). Previously, we hypothesized that the high degree of behavioural coordination and attentiveness to social partners shown by cooperative breeders would contribute to success on cooperative problem-solving tasks (Cronin et al. 2005). Indeed, cottontop tamarins were highly successful at cooperative problem solving for mutual rewards in the laboratory and showed an understanding of the role of their partner in the cooperative task (Cronin et al. 2005). Moreover, Hauser et al. (2003) have shown that cottontop tamarins discriminate between rewards received from a conspecific as a by-product of a selfish action or as an altruistic act. Tamarins were more likely to donate food to conspecifics that had donated to them altruistically.

The current series of experiments examines the effects of varied reward distributions on the cooperative behaviour of unrelated pairs of socially bonded cottontop tamarins. The dyads in this study had been housed together for at least 5 years at the onset of this study and all appeared to have a strong social bond with their partner. Unlike previous studies, we examined the effects of reciprocal reward delays extending beyond a single test session. Specifically, experiment 1 was designed to establish the tamarins' baseline performance on a mutualistic cooperative task; experiment 2 examined how the tamarins' performance changed when only one tamarin benefits from the cooperative act within a session, but the rewarded individual was alternated between sessions 
(reciprocal payoffs over days); experiment 3 assessed the tamarins' cooperative success for a single, monopolizable reward; experiment 4 measured whether cooperation persisted when only one individual in the dyad benefited from the cooperative act for the duration of the experiment.

\section{EXPERIMENT 1: MUTUALISM}

\section{Methods}

\section{Subjects}

We tested four pairs of unrelated cottontop tamarins at the University of Wisconsin, Madison. Each pair consisted of an adult female and a vasectomized male and all were subjects of our previous cooperation study (Cronin et al. 2005). The tamarins were housed in indoor cages constructed of anodized aluminium framing and polyurethane-coated steel mesh. Cages measured either $160 \times 236 \times 93 \mathrm{~cm}(\mathrm{~L} \times \mathrm{H} \times \mathrm{W})$ or $236 \times 220 \times 186 \mathrm{~cm}$. They included natural branches, wooden planks and ropes to simulate an arboreal environment. All tamarins were housed in rooms containing multiple cages but were isolated from each other visually by opaque fabric sheets. All subjects had ad libitum access to water and were not food deprived. For additional husbandry information, see Ginther et al. (2001).

\section{Apparatus}

The apparatus (Fig. 1a) was a small, clear Plexiglas box that contained two sliding trays, positioned one on top of the other with one tray protruding from either side. The apparatus measured $15 \times 13 \times 4 \mathrm{~cm}(\mathrm{~L} \times \mathrm{H} \times \mathrm{W})$ with ledges extending $20 \mathrm{~cm}$ on either side. The portion of the tray external to the apparatus on either side contained a handle to allow the subject to pull and extend the tray. The handles were $20 \mathrm{~cm}$ apart and positioned opposite each other so that a single subject could not pull both handles simultaneously. Inside the apparatus, each tray had two holes located such that when the handles were simultaneously extended the holes aligned vertically. This allowed the rewards placed in the holes on the top tray to fall to the floor of the apparatus, one reward to each subject. Elastic bands were attached to the trays causing the trays to retract immediately when the subject stopped exerting tension on the handle. Thus, both subjects had to exert tension simultaneously on the handles to align the holes (Cronin et al. 2005).

\section{Training}

All subjects of the current experiment had participated in a previous experiment with the same apparatus (Cronin et al. 2005); therefore no additional training was required.

\section{Testing procedure}

A median of 26.02 months (range 25-29, calculated as 30-day periods) elapsed since the completion of the previous cooperation experiment and the onset of the current experiment. Each pair participated in 10 sessions
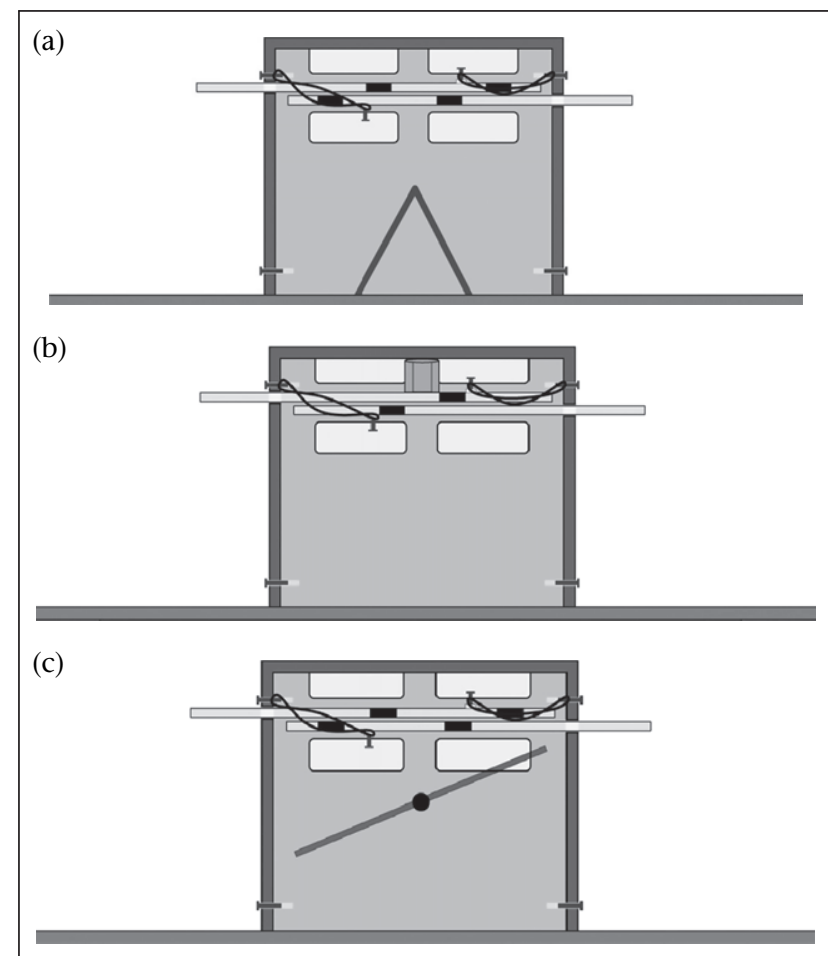

Figure 1. Side view of the apparatus. The dark portions of the trays inside the apparatus indicate holes; when the trays are simultaneously extended from opposite sides of the apparatus, the holes line up and the reward(s) fall to the floor of the apparatus. (a) The apparatus used in experiments 1 and 2 . In experiment 2 only one side of the apparatus was baited per session. (b) The apparatus used in experiment 3 . The trays contain only one hole each, and a single reward is placed within the clear cylinder equidistant from both sides of the apparatus upon baiting. (c) The apparatus used in experiment 4 . Only one side of the apparatus was baited for the duration of the experiment, and the chute inside the apparatus delivered the reward across from the individual on the baited side.

of eight trials each. All test sessions were videotaped. A test session began as soon as the experimenter finished baiting the apparatus and left the area. A test session was terminated when tamarins completed all trials or had ceased pulling handles for at least 3 min during a trial. A trial began when the apparatus was baited and ended when rewards were released from the apparatus because of simultaneous extension of the handles. The next trial began when the subjects finished eating the rewards (approximately $30 \mathrm{~s}$ ).

For all sessions, pulling behaviour at the apparatus and latencies to first pull and to solve the trial were scored from videotapes. A pull was defined as a full extension of an apparatus handle, using one or both hands, until the screw of the handle encountered the apparatus wall producing an audible click. Only full extension of the handle was scored as a pull. All pulls were recorded as occurring on the left or right side of the apparatus. Videotapes were viewed in slow motion when a pull was difficult to score. Cases in which a tamarin fully extended a handle, let it retract, then fully extended it again without removing its hands from the apparatus were scored as two separate pulls. 


\section{Data analysis}

To establish reliability, $20 \%$ of the sessions were randomly selected and coded by a second observer. Interobserver reliability was $95 \%$ with respect to pulling behaviour (animal ID, number of pulls, and side on which the pull occurred). All $P$ values reported in this and the following experiments are two tailed. Standard errors are reported following means.

The percentage of trials solved per session was calculated conservatively as the number of trials the pair solved divided by the total number of trials possible in that session had they continued to solve the trials (eight). For example, if a tamarin pair did not solve the fourth trial in a session, the session was ended and the pair was scored as having solved three of eight or $37.5 \%$ trials in that session. Percentage efficiency was calculated as the number of pulls required by a pair to solve a trial/the number of pulls executed by a pair on a trial $\times 100 \%$.

\section{Results}

\section{Task performance}

The tamarins solved a mean of $99.33 \pm 0.68 \%$ of trials $(N=307)$. In the previous cooperation study (Cronin et al. 2005), the same subjects solved $96.84 \pm 1.01 \%$ of trials $(N=612)$. The tamarins showed a mean percentage efficiency of $50.18 \pm 4.36 \%$. The percentage efficiency in our previous cooperation study (Cronin et al. 2005) with the same tamarin pairs was nearly identical, at $50.33 \pm 7.32 \%$. The tamarins' mean latency to solve the task was $15.04 \pm 3.98 \mathrm{~s}$. Again this performance measure is similar to the tamarins' previous performance on the task, $16.40 \pm 4.34 \mathrm{~s}$. None of the above performance measures differed significantly from the results presented in Cronin et al. (2005); paired $t$ tests: $P$ values $>0.10$.

\section{Side preferences}

All tamarins showed a strong side preference in this experiment, reliably pulling from one side of the apparatus or the other to solve the task $(86.9 \%$ of trials were solved when tamarins occupied their preferred side). The side preferences shown by the subjects in experiment 1 were the same preferences shown by the same pairs during the study approximately 26 months earlier.

\section{Discussion}

Experiment 1 provides updated performance measures for the tamarins on the mutualism task; these updated performance measures were necessary to enable accurate comparisons with experiments $2-4$ in which reward schemes are manipulated. The tamarins showed strong cooperative task performance for equal reward payoffs as measured by percentage of trials solved, efficiency and latency to solve the task. These performance measures were very similar to their previous experience with the task 26 months prior, showing long-term memory for the task and no need for additional training prior to additional experiments. Additionally, we confirmed the tamarins' strong side preferences, which enabled us to bias the reward distribution in the following experiment.

\section{EXPERIMENT 2: RECIPROCAL REWARDS}

We made use of the side preferences expressed by the tamarins in experiment 1 to determine whether cooperative performance would change when only one individual's preferred side was baited within a session, but the rewarded side was alternated between sessions. Because tamarins often show distress when physically separated from their mates, we decided to use the side preferences to create the desired reciprocal reward distribution rather than physically restrict their access to one side of the apparatus or the other. Although the reward distribution would have been more tightly controlled by separating the subjects and restricting the tamarins' access to the apparatus, this approach would have limited the social relevance of the task. Experiment 2 differs from previous studies of reciprocal reward payoffs (de Waal \& Berger 2000; Hauser et al. 2003; Hattori et al. 2005) in that benefits to actors are generally separated by at least 1 day, rather than balanced within a single session. Therefore, this payoff structure provides a greater temporary cost to the unrewarded cooperator.

\section{Methods}

\section{Subjects and apparatus}

The subjects and apparatus of experiment 2 were the same as those in experiment 1 .

\section{Testing procedure}

Forty-five days elapsed between the final session of experiment 1 and the first session of experiment 2. Each pair participated in 10 sessions. Each session consisted of a maximum of eight trials, with a 90-s intertrial interval (ITI). Only one side of the apparatus was baited per session. Because the apparatus was transparent, the tamarins could see from a distance which side of the apparatus was baited. The baited side was held constant on all trials within a session and alternated between sessions. As in experiment 1 , simultaneous extension of the handles from opposite sides of the apparatus was required to release the reward. However, a key difference was that simultaneous pulling only delivered a reward to the tamarin on the baited side of the apparatus whereas the other animal obtained no food. Sessions ended when tamarins completed eight trials or failed to solve a trial in $10 \mathrm{~min}$. Notice that this termination criterion differs from experiment 1 in which a trial ended if the tamarins ceased pulling handles for 3 min because a trial could continue indefinitely in the one-reward task if a tamarin pulled at the baited side only. At least $24 \mathrm{~h}$ elapsed between sessions. Time elapsed between sessions averaged 3.39 ( \pm 0.34 ) days and ranged from 1 to 11 days.

The behavioural measures used in experiment 1 were also used in experiment 2 . Additionally, we recorded the identity of the rewarded individual and whether each pull 


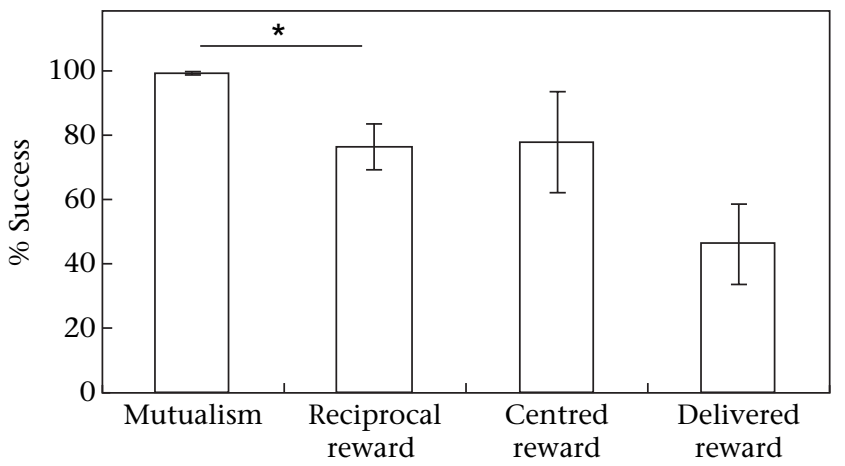

Figure 2. Percentage success (mean $\pm S E M$ ) for all experiments. Planned $t$ tests were conducted between mutualism and reciprocal reward $(P=0.041)$, reciprocal reward and centred reward $(P=0.907)$, and reciprocal reward and delivered reward $(P=0.124) .{ }^{*} P<0.05$.

was made at the baited or unbaited side. We were also interested in observing the tamarins' interactions with their partner following trials and added a 90-s ITI. During the ITI we recorded all occurrences of 32 behaviours from our laboratory's standard ethogram for social interactions using Noldus Observer Version 5.0 (Noldus Information Technology, Wageningen, The Netherlands).

\section{Data analysis}

We used paired comparisons (Student's $t$ tests, two tailed) between experiments 1 and 2 to determine whether the performance changed when only one tamarin received a reward per trial. Student's $t$ tests were chosen for between-experiment comparisons because although the sample size was small (four pairs), the $t$ test is robust to type I errors when distributions are not highly skewed (Pearson \& Please 1975). Effect sizes are included and were calculated as specified by Gibbons et al. (1993) for paired comparisons. Twenty per cent of the sessions were randomly selected and coded for reliability by a second observer. Interobserver reliability was 94\% with respect to pulling behaviour and $100 \%$ with respect to identity of the rewarded animal.

\section{Results}

\section{Task performance}

The tamarins continued to solve the task when only one reward was present, solving an average of $76.20 \pm 7.09 \%$ of trials $(N=320)$ over 10 sessions. This was a significant decrease from experiment 1 in which $99.32 \%$ of the trials were solved (paired $t$ test $t_{3}=3.449, P=0.041, d=1.725$, Fig. 2). Interestingly, all pairs solved $100 \%$ of the trials in the first one or two sessions, and then showed a sharp drop in the percentage of trials solved in the third and/ or fourth sessions. Two of the pairs then resumed their previous level of performance and continued solving $100 \%$ of the trials for the remainder of the sessions. The other two pairs solved a varied percentage of trials in the remaining sessions (Fig. 3).

To further examine the observed variation in percentage success, we analysed whether there was an effect of the number of days that had elapsed since the individual on the unbaited side had been the recipient of rewards. An analysis of variance indicated no effect of number of days elapsed between sessions on percentage success (analysis of variance, ANOVA: $\left.F_{1,34}=0.141, P=0.710\right)$.
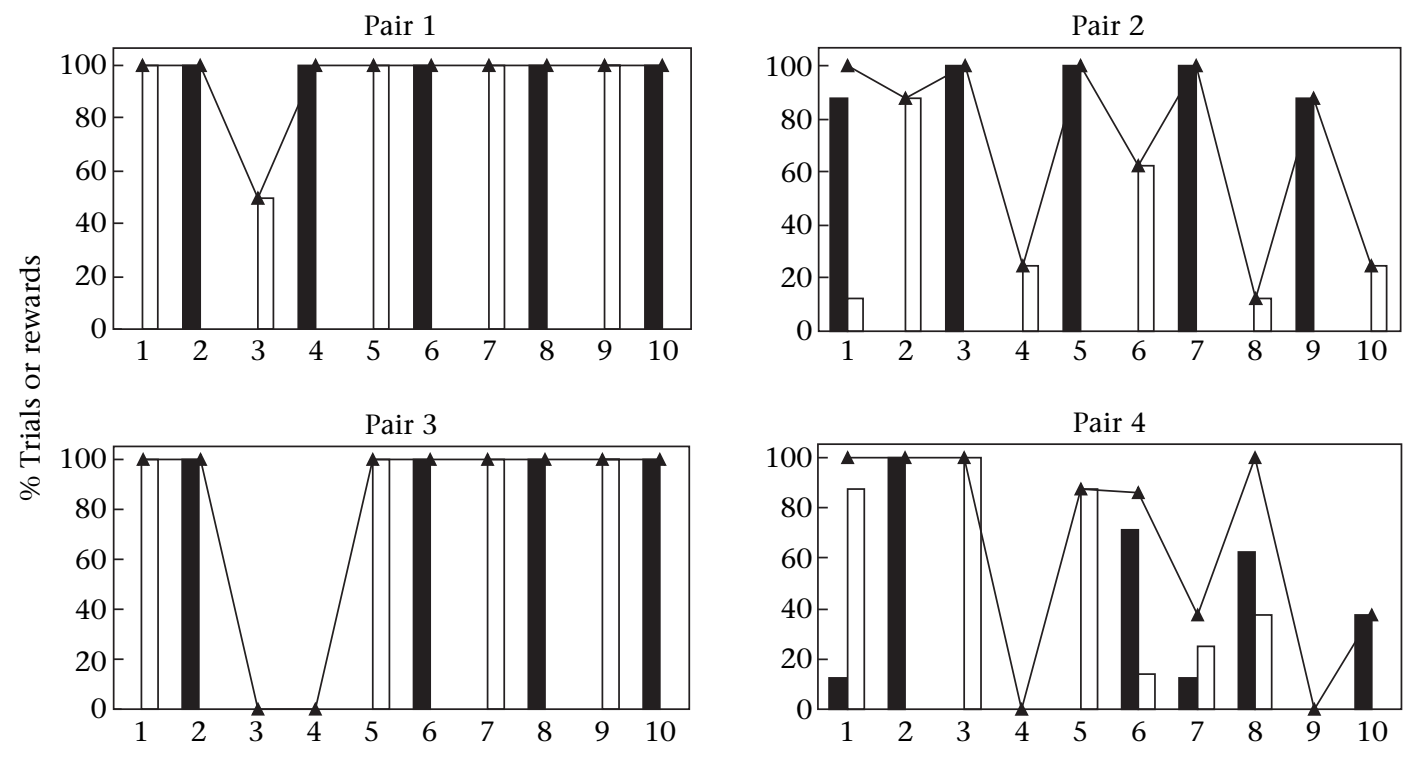

Session

Figure 3. Percentage of trials solved and percentage of rewards received in experiment 2, reciprocal reward. The line indicates the percentage of trials solved. Black bars indicate the percentage of rewards received by the female, white bars represent the percentage of rewards received by the male (percentages calculated out of total number of rewards possible each session regardless of whether the trial was solved). Pair 2 was the only pair in which one partner received significantly more rewards than the other $(P=0.010)$. Sessions in which both the male and the female received rewards indicate sessions in which the tamarins solved at least one trial on their nonpreferred side. 
Percentage efficiency in experiment 2 was $43.82 \pm 6.22 \%$, not significantly different from the efficiency shown by the tamarins in experiment 1 (paired $t$ test: $t_{3}=1.999$, $P=0.140, d=0.999)$. The tamarins took longer to solve the task, as latency to solve the task significantly increased from experiment 1 to experiment 2 , from $15.05 \pm 3.98 \mathrm{~s}$ to $24.08 \pm 4.73 \mathrm{~s}$ (paired $t$ test: $t_{3}=-6.096, P=0.009$, $d=3.048$, Fig. 4).

The tamarins made significantly more pulls per trial when the side that they currently occupied was baited than when the side that their partner occupied was baited (paired $t$ test: $t_{7}=4.7924, P=0.002, d=1.694$ ). Therefore, the tamarins pulled more often to deliver the reward to themselves than to deliver the reward to their partner.

\section{Side preferences and reward distribution}

All tamarins continued to show side preferences (97.1\% of trials were solved when tamarins occupied their preferred side). In three of the five sessions in which at least one trial was solved on a tamarin's nonpreferred side, the tamarins achieved 100\% success, thus indicating that the side preferences were not necessary for success. The side preferences shown by the subjects were the same preferences shown during experiment 1.

In three of the four pairs, reward distribution did not differ from $50 \%$ to each partner over the course of 10 sessions (binomial test: pair $1 P=0.731, N=76$, pair 3 $P=1.000, N=63$, pair $4 P=0.672, N=50)$; in pair 2 , the female received significantly more rewards than the male (binomial test: $P=0.020, N=54$ ). In this pair, the female pulled on the unbaited side less frequently than the male, in effect not allowing him as many rewards as she obtained. As expected, within each session, reward distribution was strongly biased towards the individual that preferred the side that was baited during that session, and the rewarded individual alternated between sessions (Fig. 3).

\section{Social behaviour}

Because of the low frequency of occurrence of many of the behaviours recorded during the ITI, statistical analyses of the rates of behaviours within and between the pairs

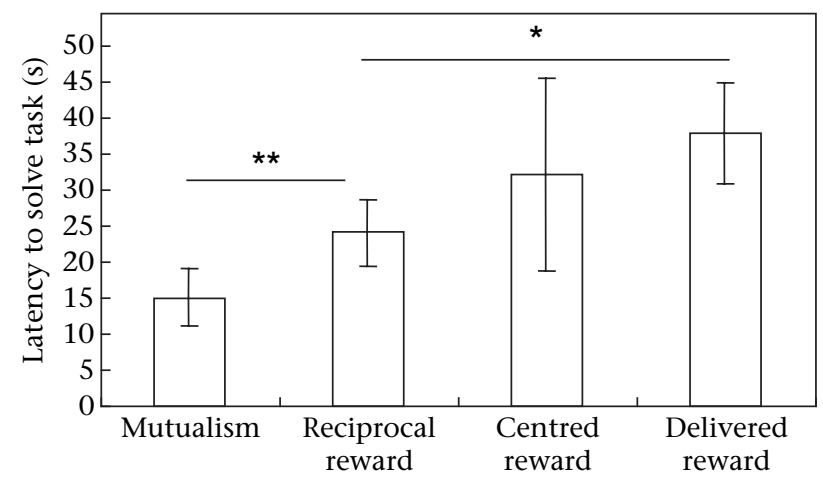

Figure 4. Latency to solve the task (mean \pm SEM) for all experiments. Planned $t$ tests were conducted between mutualism and reciprocal reward $(P=0.009)$, reciprocal reward and centred reward $(P=0.566)$, and reciprocal reward and delivered reward $(P=0.028) .{ }^{*} P<0.05$; $* * P<0.01$. were not possible. Tamarins often returned to the apparatus to await the next trial after the reward was consumed. However, aggressive behaviours directed from one tamarin to another occurred extremely rarely, despite the fact that during a session one cooperating tamarin was continuously denied rewards while their partner received rewards. Following 243 successful trials in experiment 2 (364.5 min of observation) there was one instance each of a threat face and frown at partner, three of piloerection, seven of one tamarin jumping on the other, and no instances of a tamarin biting, grabbing, or wrestling with their partner. This equates to a rate of one potentially aggressive incident every 20.3 trials. Food sharing was observed once.

\section{Discussion}

Tamarins solved significantly fewer trials and took longer to solve trials when both individuals did not benefit immediately and equally for their efforts as in experiment 1 . However, the tamarins still solved $76 \%$ of the trials. Furthermore, tamarins cooperated for reciprocal rewards separated over days, even after a delay of 11 days between sessions. In most tamarin pairs, we observed reciprocity across sessions with each tamarin receiving equal rewards over the course of the experiment. Aggression between tamarins was extremely rare, even though within most sessions only one tamarin received rewards for their cooperative efforts. Yet animals did show some selfish motivation, as evidenced by a greater rate of pulling when the side they occupied would be rewarded and lack of food sharing with their partner. One limitation of this experiment was that the side preferences, although they enabled the reciprocal reward distribution, did not allow us to analyse how the tamarins would respond to a situation in which a single, monopolizable reward is produced from the cooperative act but the individual who will ultimately gain access to the reward is unknown to the actors prior to cooperating. Experiment 3 was designed to address this question.

\section{EXPERIMENT 3: CENTRED REWARD}

In experiment 3 , we modified the apparatus design so that a single, monopolizable reward was delivered equidistant from both subjects upon completion of the cooperative act, and the identity of the rewarded animal could not be known before the tamarins engaged in cooperation.

\section{Methods}

\section{Subjects and apparatus}

The subjects of experiment 3 were the same as those in experiments 1 and 2 . For experiment 3, the apparatus was modified such that the trays within the apparatus had only a single, centred hole. A clear cylindrical piece was added to the inside of the apparatus to ensure that when baited, the single reward rested atop the trays equidistant from both subjects. When the trays were simultaneously extended from opposite sides of the apparatus, the reward 
fell to the centre of the apparatus floor. The triangular piece of Plexiglas on the floor of the apparatus was removed, and two small pieces of Plexiglas were secured to ensure that the reward remained at the centre and did not roll towards one subject (Fig. 1b).

\section{Testing procedure}

Seven months elapsed between the final session of experiment 2 and the first session of experiment 3. Each pair participated in 10 sessions, except for one pair (pair 3) that participated in nine sessions because of a problem with the apparatus during one session. Each session consisted of a maximum of eight trials. Sessions ended when tamarins either completed all eight trials or failed to solve a trial in $10 \mathrm{~min}$. The next session occurred as scheduled. At least $24 \mathrm{~h}$ elapsed between sessions with an average of $1.81 \pm 0.22$ days between sessions and a range of 1-5 days. There was a 90-s ITI and sessions were videotaped and scored as in experiment 2. During video scoring of experiment 3 we also scored whether each subject made an attempt to retrieve the reward from the centre of the apparatus floor upon solving the task. A 'grab' was scored whenever any part of the tamarin's body crossed the side plane of the apparatus into the bottom of the apparatus where the food reward was located.

\section{Data analysis}

Paired comparisons of experiments 2 and 3 were used to determine whether the tamarins' performance changed between the condition during which one tamarin was guaranteed to receive a reward and the other was not versus the condition in which neither tamarin was guaranteed a reward for the cooperative act. Twenty per cent of the sessions were randomly selected and coded for reliability by a second observer. Interobserver reliability was $96 \%$ with respect to pulling behaviour and 100\% with respect to identity of the rewarded animal.

\section{Results}

\section{Task performance}

The tamarins continued to solve the task when a single, centred reward was present, solving an average of $77.71 \pm 15.68 \%$ of the trials $(N=304)$. This was not significantly different from the percentage of trials solved in experiment 2 (paired $t$ test: $t_{3}=-0.1275, P=0.907$, $d=0.064)$, however, the pairs displayed more variation in experiment 3 (Fig. 2).

Percentage efficiency was $58.57 \pm 10.45 \%$, not significantly different from the efficiency shown by the tamarins in experiment 2 (paired $t$ test: $t_{3}=-2.162, P=0.119$, $d=1.081$ ). Latency to solve the task also did not differ significantly from experiment 2 to experiment 3; latency to solve the task in experiment 2 was $24.08 \pm 4.73 \mathrm{~s}$ and in experiment 3 was $32.12 \pm 13.35 \mathrm{~s}$ (paired $t$ test: $t_{3}=-0.6423, P=0.566, d=0.321$, Fig. 4$)$.

\section{Side preferences and reward distribution}

All tamarins continued to show side preferences $(91.5 \%$ of trials were solved when tamarins occupied their preferred side). The side preferences shown by the subjects in experiment 3 were the same preferences shown during experiments 1 and 2 .

In three of the four pairs, rewards received by each partner did not differ significantly from 50\% (binomial test: pair 2: $P=0.813, N=71$; pair $3: P=0.162, N=62$; pair $4: P=0.690, N=25)$; in pair 1 the male received significantly more rewards than the female (binomial test: $P=0.050, N=76$ ). In contrast to experiment 2 in which reward distribution was unequal within a session but equal across sessions, reward distributions were generally equal within sessions (Fig. 5).

We analysed the subjects' attempts to retrieve the reward once the task was solved to determine whether the reward distribution was a result of the tamarins actively deferring rewards to their partners, or simply due to chance. We first verified that within each pair, the subjects had equal chances of obtaining the reward if they both grabbed for it. Chi-squared analyses indicated that within each pair, on trials when both subjects grabbed for the reward, the distribution of rewards did not significantly differ from $50 \%$ to each subject (pair $1: \chi_{1}^{2}=0.472$, $P=0.492$; pair $2: \chi_{1}^{2}=1.473, P=0.225$; pair $3: \chi_{1}^{2}=0.348$, $P=0.56$; pair $\left.4: \chi_{1}^{2}=2.579, P=0.108\right)$.

The percentage of trials on which an individual did not grab ranged from $6.58 \%$ to $23.68 \%(\bar{X} \pm \mathrm{SE}=12.45 \pm$ $2.19 \%)$. The tamarin that showed the greatest percentage of trials without grabbing was the same individual (Sc) that received significantly fewer rewards than her partner (In). Seventy-two per cent of the trials on which the female did not grab for the reward were in the first half of sessions. It appears that the unequal reward distribution in this pair may be attributed to the female learning more slowly than her partner to reach inside the apparatus for the single reward. In the three pairs that showed reward distributions not significantly different from 50\% to each actor, both tamarins grabbed for the reward on $88 \%$ of trials. Thus, the equal reward distribution did not appear to be facilitated by individuals deferring rewards to their partners, but rather by the equal chance that the tamarins would retrieve the reward given that they both grabbed for it.

\section{Social behaviour}

After a trial was solved the tamarins were forced to scramble for the reward that was delivered to the centre of the apparatus floor. Even in this highly competitive situation, aggression was nearly absent. Following 234 successful trials (351 min of observation) there was one instance each of a bite and wrestle, two of piloerection and grab at partner, and no instances of a tamarin delivering a threat face towards, jumping on or frowning at their partner. This equates to a rate of one potentially aggressive incident every 39 trials. There were no instances of food sharing in experiment 3.

\section{Discussion}

Tamarins continued to cooperate and show high success and efficiency for a single, monopolizable reward. Reward distribution did not significantly differ from 50\% to each 

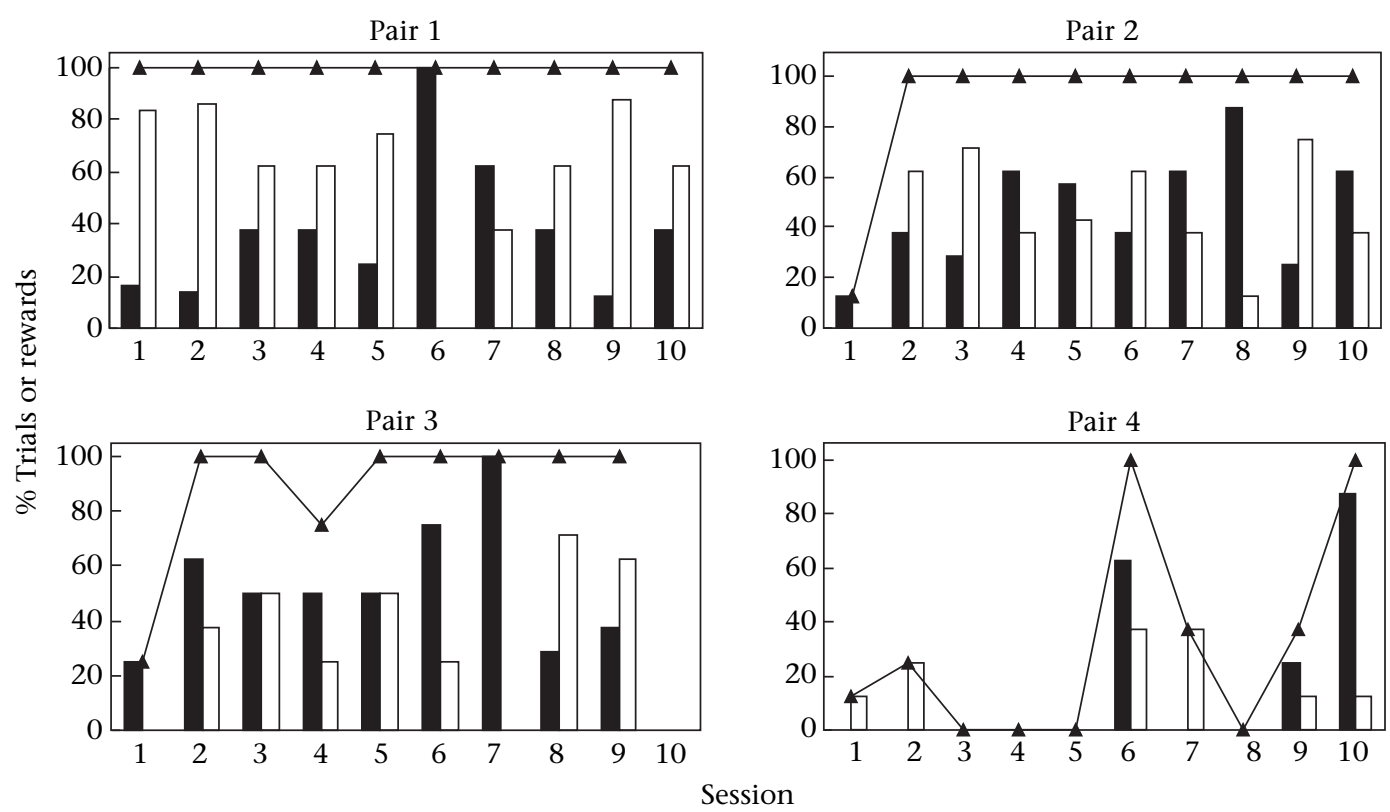

Figure 5. Percentage of trials solved and percentage of rewards received in experiment 3, centred reward. The line indicates the percentage of trials solved. Black bars indicate the percentage of rewards received by the female (percentage calculated out of total number of rewards possible each session regardless of whether the trial was solved), white bars represent the percentage of rewards received by the male. Pair 1 was the only pair in which one partner received significantly more rewards than the other $(P=0.025)$.

actor in three of the four pairs; however, this did not appear to be because of any active effort by the subjects to minimize inequality. As in experiment 2 , aggression was virtually absent and individuals continued to participate even though they were required to compete for the reward upon completion of the cooperative act.

\section{EXPERIMENT 4: DELIVERED REWARD}

Experiments 1-3 provide evidence that tamarins will cooperate when rewards are equalized over time, whether they are immediately equal after each cooperative act (experiment 1 ), equal across sessions/days (experiment 2 ), or equal within a session/day (experiment 3 ). However, we do not know to what extent cooperative behaviour would be maintained if the reward distribution to actors remains unequal throughout the duration of the experiment. The aim of experiment 4 was to determine whether the tamarins would continue to cooperate when the same actor was repeatedly rewarded and the other actor received nothing.

\section{Methods}

\section{Subjects and apparatus}

The subjects of experiment 4 were the same as those in experiments 1, 2 and 3. For experiment 4, the apparatus was similar to the apparatus used in experiment 2; that is, the trays had two reward locations, one near each tamarin. However, in this experiment, one side of the apparatus was baited for the duration of the experiment. Additionally, a diagonal chute was added that, upon simultaneous extension of the trays, caused the reward located near one tamarin to be delivered to the tamarin at the other side of the apparatus (Fig. 1c).

\section{Testing procedure}

The side of the apparatus to be baited was determined randomly for each pair before the onset of the experiment. This resulted in two pairs for which the male's preferred side was baited and rewards were delivered to the female's preferred side, and two pairs for which the female's preferred side was baited and rewards were delivered to the male's preferred side. Note that the subject on the unbaited side would always receive the reward because of the design of the apparatus.

Nine months elapsed between the final session of experiment 3 and the first session of experiment 4 . Each session consisted of a maximum of eight trials, and the subjects participated in 10 sessions. Sessions ended when tamarins either completed all eight trials or failed to solve a trial in $10 \mathrm{~min}$. The next session occurred as scheduled. At least $24 \mathrm{~h}$ elapsed between sessions. Time elapsed between sessions averaged $1.22( \pm 0.11)$ days and ranged from 1 to 3 days. There was a 90-s ITI and sessions were videotaped and scored in the same manner as in experiment 3, with the exception of 'grab', which was no longer relevant.

\section{Data analysis}

Paired comparisons of experiments 2 and 4 were used to determine whether the tamarins' performance changed between the condition in which both tamarins pulled to release a reward to the tamarin on the baited side, and a condition in which both tamarins pulled but one tamarin was delivering a reward to the tamarin on the 
opposite side of the apparatus. Twenty per cent of the sessions were randomly selected and coded for reliability by a second observer. Interobserver reliability was $90 \%$ with respect to pulling behaviour and 100\% with respect to the identity of the rewarded animal.

\section{Results}

\section{Task performance}

Percentage of trials solved in experiment 4 decreased to $46.25 \pm 12.45 \%$. This percentage of trials solved was not significantly different from the percentage of trials solved in experiment 2 (paired $t$ test: $t_{3}=2.125, P=0.124$, $d=1.063$, Fig. 2). Failure to reject the null hypothesis of no change in percentage of trials solved should be interpreted cautiously, as the power to detect the large effect size observed here is only 0.317 because of the small sample size in this study. The pairs showed less consistent trial solving than in previous experiments; for example, two pairs did not solve any trials for five continuous sessions, one pair oscillated between solving the majority of trials and solving very few, and the final pair showed a relatively consistent decrease in percentage of trials solved over sessions (Fig. 6).

Percentage efficiency was $43.79 \pm 9.66 \%$. This percentage efficiency was not significantly different from the percentage efficiency shown in experiment 2 (paired $t$ test: $\left.t_{3}=0.003, P=0.998, d=0.002\right)$. Latency to solve the task increased significantly between experiment 2 and experiment 4 , from $24.08 \pm 4.73 \mathrm{~s}$ to $37.86 \pm 7.04 \mathrm{~s}$ (paired $t$ test: $t_{3}=-4.009, P=0.028, d=2.004$, Fig. 4 ).

The reward was delivered to the tamarin away from the tamarin that pulled the handle closest to the reward.
This was the opposite of the reward scheme used in experiment 2 . The tamarins appeared to learn this reward scheme during the course of the experiment. Pair 4 virtually stopped responding after session 5 (Fig. 6). Of the remaining pairs, the average percentage of trials in which the first pull was made on the donor's side of the apparatus was $65.02 \pm 14.56 \%$ in sessions $1-5$, whereas it was only $20.70 \pm 2.07 \%$ in sessions $6-10$. Because the tamarins adhered strongly to their side preferences $(94.79 \pm 0.02 \%$ of all pulls were made on the preferred side), the tamarin preassigned as the donor tamarin initiated more trials in early sessions than in later sessions.

\section{Side preferences and reward distribution}

All tamarins continued to show side preferences $(97.3 \%$ of trials were solved when tamarins occupied their preferred side). The side preferences shown by the subjects were the same preferences shown during previous experiments.

Reward distribution in experiment 4 was highly biased towards the individual on the unbaited side, as designed. Two animals received $100 \%$ of the rewards, one received 94\% and one 93\% (Fig. 6). Reward distribution differing from $100 \%$ to a single actor indicates at least one trial on which side preferences were reversed.

\section{Social behaviour}

Following all trials of experiment 4, only one individual received a reward after both exerted effort to solve the task. However, as in the previous experiments, aggression was rare. Following 148 successful trials (222 min of observation) there was one instance each of an animal displaying piloerection, grabbing and frowning at their partner.
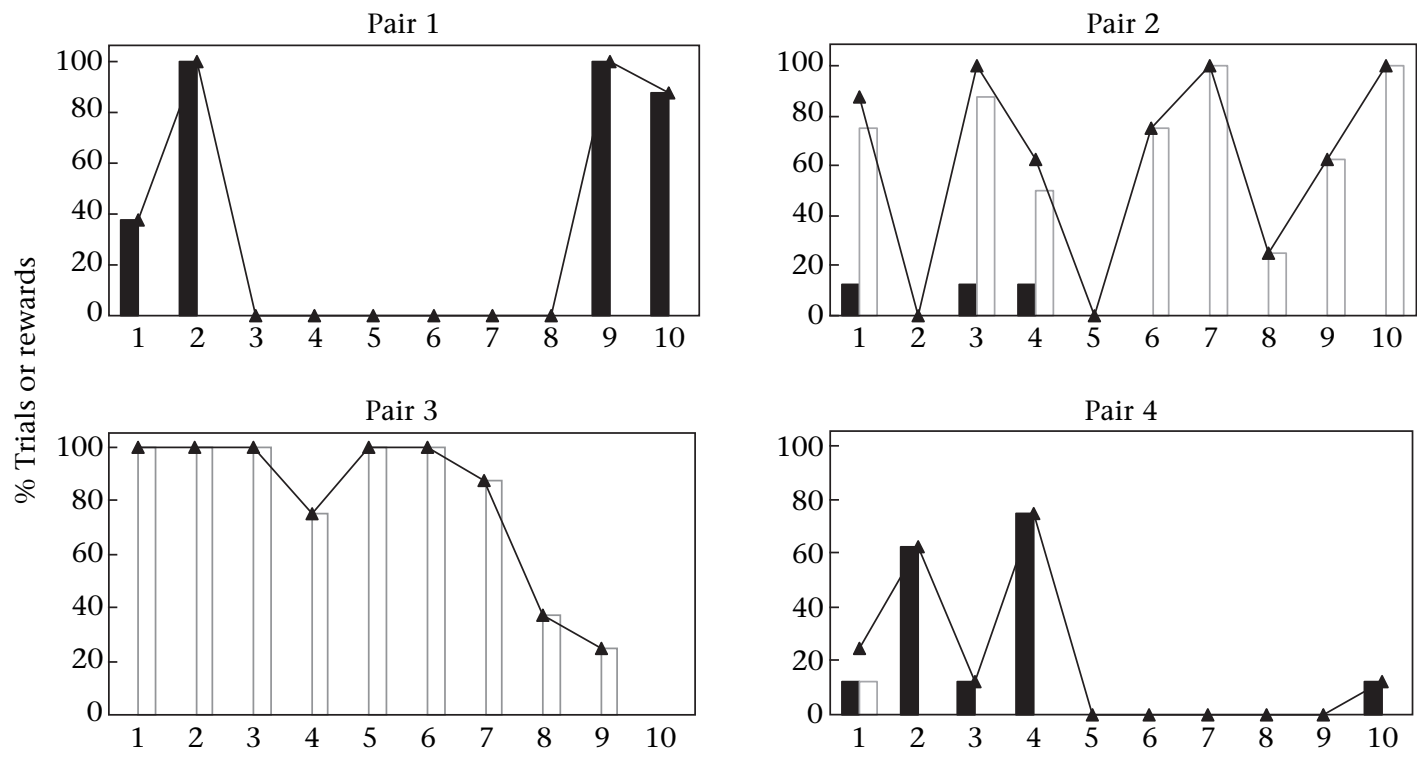

Session

Figure 6. Percentage of trials solved and percentage of rewards received in experiment 4, delivered reward. The line indicates the percentage of trials solved. Black bars indicate the percentage of rewards received by the female (percentage calculated out of total number of rewards possible each session regardless of whether the trial was solved), white bars represent the percentage of rewards received by the male. In all pairs one individual received more rewards than their partner. Sessions in which both the male and the female received rewards indicate sessions in which the tamarins solved at least one trial on their nonpreferred side. 
There were no instances of biting, wrestling, displaying a threat face, or jumping on their partner. This equates to a rate of one potentially aggressive incident every 49.3 trials. There were no instances of food sharing in experiment 4 .

\section{Discussion}

Experiment 4 examined whether the tamarins would continue to cooperate when the same actor was repeatedly rewarded and the other actor received nothing. We found that the tamarins solved $46 \%$ of trials in this condition. This is a nonsignificant decrease from experiment 2 in which the rewarded individual was alternated between sessions (76\% of trials solved). However, the inconsistent performance of the pairs (Fig. 6) indicates that the delivered reward may be a less conducive environment to cooperation and the nonsignificant finding may be because of lack of power in this study. The tamarins showed a significantly longer latency to solve the task in the delivered reward experiment compared with the mutual reward experiment. The transition from the donor to the recipient initiating the majority of first pulls of each trial indicates that the tamarins learned the new reward scheme during the experiment, yet this does not cause cooperative behaviour of the donor to extinguish in the following sessions (Fig. 6).

\section{GENERAL DISCUSSION}

Overall, we found that the tamarins' cooperative performance was sensitive to the rewards they would receive following their cooperative effort. When actors worked for mutual rewards (experiment 1), their cooperative performance was highest, nearing $100 \%$. When the distribution of rewards was altered to provide reciprocal reward payoffs to the actors, the tamarins' cooperative success significantly decreased to $76 \%$, showing sensitivity to the reciprocal reward scheme. However, it should be noted that percentage success in this study was calculated conservatively (if a pair did not solve a trial mid-session in the maximum time allotted, the session was ended and they were scored as failing all remaining trials in that session as well). Additionally, we do not know whether cooperation would have recommenced within a session if sessions were not ended after the maximum trial length was reached. Direct comparisons with other species are not possible since no other species have been tested on the apparatus used in these experiments, however, cooperative success of $76 \%$ is higher than or comparable with other cooperative problem-solving experiments with nonhuman primates with single or mutual rewards (capuchins, Cebus apella: Mendres \& de Waal 2000; de Waal \& Berger 2000; de Waal \& Davis 2003; Hattori et al. 2005; Brosnan et al. 2006; chimpanzees: Melis et al. 2006a).

The tamarins solved a similar percentage of trials in experiments 2 and 3, indicating that the tamarins may be equally successful at solving the cooperative task when there is a single monopolizable reward present, regardless of whether the individual to be rewarded is identified prior to the cooperative act (experiment 2) or following the cooperative act (experiment 3). Experiment 4 was the only experiment that did not provide the tamarins with equal reward distribution over repeated interactions, and in this situation the tamarins performed least well with the longest latency to solve the task and the lowest percentage of trials solved. However, they still solved nearly half of the trials. The results of this series of experiments indicate that tamarins continue to cooperate when not equally rewarded, but are sensitive to reward distributions and will cooperate most often when both individuals receive rewards either simultaneously or over repeated interactions.

Even when several days elapsed between the exchange of donor and recipient roles in the reciprocal reward experiment (experiment 2) the tamarins solved the majority of trials. We believe the tamarins' success on this delayed reciprocity task was most likely due to what de Waal \& Luttrell (1988) have termed 'symmetry based reciprocity', an explanation for reciprocity that does not require any complex cognitive score-keeping of past interactions. Symmetry-based reciprocity is characterized by 'exchanges between closely bonded individuals who help each other without stipulating equivalent returns' (de Waal \& Luttrell 1988, p. 103). Given the long-term pair bonds between the tamarins in this study, their success could likely be explained by this type of reciprocity. Characteristically, the tamarin dyads in this study have shown no signs of aggression, slept together nightly, huddled with and groomed each other during the day, and fed simultaneously in proximity with each other. Callitrichids are strongly interdependent on each other for survival and reproductive success. Their relationships have been described as valuable and secure, with aggression being very rare (Schaffner \& Caine 2000; Aureli \& Schaffner 2006). Therefore, perhaps the simplest explanation for the tamarins' success on the reciprocal reward task is that they do not 'keep score' with each other, and isolated asymmetric interactions are relatively unimportant in the larger context of their relationship.

Results of many studies indicate that the nature of the relationship between actors will play a role in their cooperative success. Brosnan et al. (2005) have shown that chimpanzees with long-term relationships are more tolerant of inequitable reward distributions. Also, Beck (1973) found that the only two hamadryas baboons, Papio hamadryas, in a captive group to show cooperative tool use were two individuals in a bonded pair. Recent evidence suggests that while chimpanzees in long-term adult-adult pairs interact reciprocally with each other, reciprocity does not persist similarly in mother-offspring pairs ( $\mathrm{S}$. Yamamoto \& M. Tanaka, unpublished data). Melis et al. (2006b) showed that pairs of chimpanzees that were more likely to share food outside of the testing situation showed greater success on a cooperative task, indicating that the nature of the relationship between actors will play a role in their success. These observations indicate that the nature of the relationship between actors may affect their cooperative performance and tolerance for temporarily unequal reward distributions. 
Although rates of aggression were extremely low throughout all single reward conditions, the rate of aggression was highest during the first exposure to a condition in which tamarins were not equally rewarded (experiment 2). Rates of aggression were then lower during the competitive experiment (experiment 3 ), and the lowest during experiment 4, in which one animal was repeatedly unrewarded. Therefore, the aggressive encounters observed may have stemmed from the tamarins' adjustment to the schemes involving one reward after having repeatedly received mutual rewards rather than a direct response to the competition with their partner or unfair reward distribution. These findings are similar to those of Rapaport (1998) in which aggression in golden lion tamarins, Leontopithecus rosalia, was reliably predicted by search time during a foraging task but not by reward abundance. Notably, aggression by the golden lion tamarins was very infrequent, as it was in the current study.

The design of experiment 4 biased the reward distribution such that the same individual was likely to be rewarded after every cooperative act for the duration of the experiment. In one pair (pair 3) we observed decreasing cooperative success as sessions proceeded, the pattern we expected to find if the unrewarded tamarin became increasingly unwilling to cooperate without any payoff. However, the other three pairs did not display this pattern of decline, appearing to cooperate sporadically, with two pairs failing to cooperate for five or more consecutive sessions before resuming cooperation. There are many potential reasons for their varied performance. One possible explanation is that the unrewarded individuals are willing to cooperate, just not as often as when they received half the rewards. Alternatively, the unrewarded individuals could have been cooperating sporadically to investigate whether the reward scheme had changed. Given that the subjects had been through many different reward scenarios by the time they participated in experiment 4, they may have been sampling the experiment to determine whether it would benefit them to resume cooperation. Finally, Schuster \& Perelberg (2004) have suggested that cooperation may be intrinsically rewarding as a social interaction. This perspective may elucidate why individuals may choose to cooperate when their behaviours cannot be explained entirely by tangible rewards.

Noe (2006) recently suggested that the tamarins' previous cooperative performance in the mutualism task (Cronin et al. 2005) may be explained by conditioning; specifically that the tamarins learned that pulling in the presence of their partner led to a reward. Although the conditioned response explanation might account for the tamarins' performance in the experiments in which both individuals were rewarded for coordinating their pulling behaviour (Cronin et al. 2005, experiment 1 this study), the conditioned response explanation cannot account for the tamarins' performance in experiments 2-4. In experiment 2, the tamarins adjusted their rate of pulling based on whether the reward would be delivered to them or to their partner, yet they continued pulling in both situations and solved the majority of trials, even though their reduced pulling behaviour indicates that they learned they were unlikely to receive a reward when they occupied the unbaited side. In experiment 2 , the pairs showed a decline in success after the first few sessions, and then a subsequent reinstatement of success (two pairs) or variable success (two pairs) over the remaining sessions. If the tamarins' performance could be explained by a simple conditioned response to pull in the presence of the partner, we would expect to see consistently high performance across all sessions of experiment 2 and experiment 3 , especially since learning theory predicts that variable schedules of reinforcement lead to more persistent behaviour (Hilgard \& Bower 1966). In experiment 4, we saw that the tamarins' motivation to pull was based not only on the presence or absence of the partner but also the likelihood that they would be rewarded. In these experiments, the tamarins were not showing an automatic response but rather attending to the different reward scenarios presented in each experiment. Additionally, in all of the above experiments there was evidence for some selfish motivation, but their performance cannot be explained by selfish motivation alone.

A limitation of this study is that the effect of previous cooperative experience cannot be determined. All subjects in this study were first exposed to a cooperative task in which both individuals received rewards, and then subsequently presented with the reciprocal, centred and delivered reward scheme in that order. We cannot determine from this series of experiments whether the tamarins would perform differently had they experienced the reward schemes in a different order than presented here.

The results of these studies therefore show that cottontop tamarins coordinate their actions and cooperate even in situations in which they will not be immediately or equally rewarded for their efforts. However, the tamarins also showed some selfish motivation and cooperated most consistently for equal rewards. The pair-bonded tamarins rarely to never showed any aggressive behaviours towards one another following an inequitable distribution of rewards. The cooperative social system of callitrichids and the strong pair bonds between the dyads in this study may contribute to their success on these tasks in comparison with more competitive species or dyads without strong social relationships.

\section{Acknowledgments}

This research was supported by NIMH MH029775 and a National Science Foundation Graduate Research Fellowship to K.A.C. The authors would like to thank Rosamunde Almond and Matthew Campbell for advice and critical feedback, Jennifer Gaudio, Emily Rothwell and Kendra Svehlek for assistance in data collection and coding, Gustl Anzenberger for stimulating conversation that led to the development of the final experiment in this report, two anonymous referees whose feedback improved this manuscript, Andrew Mulder for construction of the 
apparatus, Bob Becker for the diagram of the apparatus, and the staff of the Snowdon laboratory.

\section{References}

Aureli, F. \& Schaffner, C. 2006. Causes, consequences and mechanisms of reconciliation: the role of cooperation. In: Cooperation in Primates and Humans: Mechanisms and Evolution (Ed. by P. M. Kappeler \& C. P. van Schaik), pp. 121-136. Berlin, Heidelberg: Springer-Verlag.

Beck, B. 1973. Cooperative tool use by captive hamadryas baboons. Science, 182, 594-597.

Boesch, C. \& Boesch, H. 1989. Hunting behavior of wild chimpanzees in the Tai National Park. American Journal of Physical Anthropology, 78, 547-573.

Brosnan, S. F. \& de Waal, F. B. M. 2003. Monkeys reject unequal pay. Nature, 425, 297-299.

Brosnan, S. F. \& de Waal, F. B. M. 2006. Partial support from a nonreplication: comment on Roma, Silberberg, Ruggiero, and Suomi (2006). Journal of Comparative Psychology, 120, 74-75.

Brosnan, S. F., Schiff, H. C. \& de Waal, F. B. M. 2005. Tolerance for inequity may increase with social closeness in chimpanzees. Proceedings of the Royal Society of London, Series B, 272, 253-258.

Brosnan, S. F., Freeman, C. \& De Waal, F. B. M. 2006. Partner's behavior, not reward distribution, determines success in an unequal cooperative task in capuchin monkeys. American Journal of Primatology, 68, 713-724.

Burton, J. J. 1977. Absence of spontaneous cooperative behavior in a troop of Macaca fuscata confronted with baited stones. Primates, 18, 359-366.

Caine, N. G. 1993. Flexibility and co-operation as unifying themes in Saguinus social organization and behaviour: the role of predation pressures. In: Marmosets and Tamarins: Systematics, Behaviour and Ecology (Ed. by A. B. Rylands), pp. 200-219. New York: Oxford University Press.

Chalmeau, R. 1994. Do chimpanzees cooperate in a learning task? Primates, 35, 385-392.

Chalmeau, R. \& Gallo, A. 1996. What chimpanzees (Pan troglodytes) learn in a cooperative task. Primates, 37, 39-47.

Chalmeau, R., Lardeux, K., Brandibas, P. \& Gallo, A. 1997. Cooperative problem solving by orangutans (Pongo pygmaeus). International Journal of Primatology, 18, 23-32.

Coussi-Korbel, S. \& Fragaszy, D. M. 1995. On the relation between social dynamics and social learning. Animal Behaviour, 50, 1441.

Cronin, K. A., Kurian, A. V. \& Snowdon, C. T. 2005. Cooperative problem solving in a cooperatively breeding primate (Saguinus oedipus). Animal Behaviour, 69, 133-142.

Dugatkin, L. A. 1997. Cooperation among Animals. An Evolutionary Perspective. Oxford: Oxford University Press.

Fady, J. C. 1972. Absence de cooperation de type instrumental en milieu naturel chez Papio papio. Behaviour, 93, 157-164.

Fehr, E. \& Fischbacher, U. 2003. The nature of human altruism. Nature, 435, 785-791.

Gibbons, R. D., Hedeker, D. R. \& Davis, J. M. 1993. Estimation of effect size from a series of experiments involving paired comparisons. Journal of Educational Statistics, 18, 271-279.

Ginther, A. J., Ziegler, T. E. \& Snowdon, C. T. 2001. Reproductive biology of captive male cottontop tamarin monkeys as a function of social environment. Animal Behaviour, 61, 65-78.

Hamilton, W. D. 1964. The genetical evolution of social behavior. Journal of Theoretical Biology, 7, 1-52.

Hare, B. \& Tomasello, M. 2004. Chimpanzees are more skilful in competitive than in cooperative cognitive tasks. Animal Behaviour, $68,571-581$.
Hattori, Y., Kuroshima, H. \& Fujita, K. 2005. Cooperative problem solving by tufted capuchin monkeys (Cebus apella): spontaneous division of labor, communication, and reciprocal altruism. Journal of Comparative Psychology, 119, 335-342.

Hauser, M. D., Chen, K. M., Chen, F. \& Chuang, E. 2003. Give unto others: genetically unrelated cotton-top tamarin monkeys preferentially give food to those who altruistically give food back. Proceedings of the Royal Society of London, Series B, 23632370.

Hilgard, E. R. \& Bower, G. H. 1966. Skinner's operant conditioning In: Theories of Learning (Ed. by R. M. Elliott, G. Lindzey \& K. MacCorquodale), pp. 107-145. New York: Merideth.

Jensen, K., Hare, B., Call, J. \& Tomasello, M. 2006. What's in it for me? Self-regard precludes altruism and spite in chimpanzees. Proceedings of the Royal Society of London, Series B, 273, 1013-1021.

Melis, A. P., Hare, B. \& Tomasello, M. 2006a. Chimpanzees recruit the best collaborators. Science, 311, 1297-1300.

Melis, A. P., Hare, B. \& Tomasello, M. 2006b. Engineering cooperation in chimpanzees: tolerance constraints on cooperation. Animal Behaviour, 72, 275.

Mendres, K. A. \& de Waal, F. B. M. 2000. Capuchins do cooperate: the advantage of an intuitive task. Animal Behaviour, 60, 523-529.

Mesterton-Gibbons, M. \& Dugatkin, L. A. 1997. Cooperation and the Prisoner's Dilemma: towards testable models of mutualism versus reciprocity. Animal Behaviour, 54, 551-557.

Noe, R. 2006. Cooperation experiments: coordination through communication versus acting apart together. Animal Behaviour, 71, 1-18.

Pearson, E. S. \& Please, N. W. 1975. Relation between the shape of population distribution and the robustness of four simple test statistics. Biometrika, 62, 223-241.

Petit, O., Desportes, C. \& Thierry, B. 1992. Differential probability of 'coproduction' in two species of macaque (Macaca tonkeana, M. mulatta). Ethology, 90, 107-120.

Rapaport, L. G. 1998. Optimal foraging theory predicts effects of environmental enrichment in a group of adult golden lion tamarins. Zoo Biology, 17, 231-244.

Roma, P. G., Silberberg, A., Ruggiero, A. M. \& Suomi, S. J. 2006. Capuchin monkeys, inequity aversion, and the frustration effect. Journal of Comparative Psychology, 120, 67-73.

Sachs, J. L., Mueller, U. G., Wilcox, T. P. \& Bull, J. J. 2004. The evolution of cooperation. Quarterly Review of Biology, 79, 135-160.

Schaffner, C. \& Caine, N. G. 2000. The peacefulness of cooperatively breeding primates. In: Natural Conflict Resolution (Ed. by F. Aureli \& F. B. M. de Waal), pp. 155-169. Berkeley: University of California Press.

van Schaik, C. P. \& Kappeler, P. M. 2006. Cooperation in primates and humans: closing the gap. In: Cooperation in Primates and Humans: Mechanisms and Evolution (Ed. by P. M. Kappeler \& C. P. van Schaik), pp. 3-21. Berlin: Springer-Verlag.

Schuster, R. \& Perelberg, A. 2004. Why cooperate? An economic perspective is not enough. Behavioral Processes, 66, 261-277.

Silk, J.B. In press. The strategic dynamics of cooperation in primate groups. Advances in the Study of Behavior, 37.

Silk, J. B., Brosnan, S. F., Vonk, J., Henrich, J., Povinelli, D. J., Richardson, A. S., Lambeth, S. P., Mascaro, J. \& Schapiro, S. J. 2005. Chimpanzees are indifferent to the welfare of unrelated group members. Nature, 437, 1357-1359.

Snowdon, C. T. 1996. Parental care in cooperatively breeding species. In: Parental Care: Evolution, Mechanisms and Adaptations (Ed. by J. S. Rosenblatt \& C. T. Snowdon), pp. 643-689. San Diego: Academic Press.

Tebbich, S., Taborsky, M. \& Winkler, H. 1996. Social manipulation causes cooperation in keas. Animal Behaviour, 52, 1-10. 
Tomasello, M. \& Call, J. 1997. Social knowledge and interaction. In: Primate Cognition, pp. 191-230. Oxford: Oxford University Press.

Trivers, R. L. 1971. The evolution of reciprocal altruism. Quarterly Review of Biology, 46, 35-57.

de Waal, F. B. M. \& Berger, M. L. 2000. Payment for labour in monkeys. Nature, 404, 563. de Waal, F. B. M. \& Davis, J. M. 2003. Capuchin cognitive ecology: cooperation based on projected returns. Neuropsychologia, 41, 221-228.

de Waal, F. B. M. \& Luttrell, L. M. 1988. Mechanisms of social reciprocity in three primate species: symmetrical relationship characteristics or cognition? Ethology and Sociobiology, 9, 101-118.

Werdenich, D. \& Huber, L. 2002. Social factors determine cooperation in marmosets. Animal Behaviour, 64, 771-781. 